\title{
Shapes of Miura Mesh Mechanism with Mobility One
}

Valentina Beatini ${ }^{1, *}$ and Koray Korkmaz ${ }^{2}$

\author{
${ }^{1}$ Post Ph.D., Arch., valentinabeatini@iyte.edu.tr \\ ${ }^{2}$ Prof. Arch., koraykorkmaz@iyte.edu.tr \\ Department of Architecture, Izmir Institute of Technology, Turkey
}

(Submitted on 18/9/2012, Reception of revised paper 16/4/2013, Accepted on 6/7/2013)

\begin{abstract}
The Miura Ori is a mechanism composed by a polyhedral deployable surface. It has favorable qualities from engineering prospective that lead the growing interest on it. The present work focuses on its transmission of motion. The mechanism can be represented by spherical 4 bar linkages, and on this account a simple and effective mobility formula is presented. The mechanism having a number of excessive rigid members, it is also possible to remove all or some of them, variously arranged. The changes are included in the calculation of allowable mobility of the system. The resulting tool can be directly used for the design of deployable Miura Ori surfaces with customized shape.
\end{abstract}

Key Words: deployable, Miura Ori, mobility, folded plates, customized kinetic meshes, kinetic architecture, flexible meshes

\section{INTRODUCTION}

\subsection{The Miura Orimap}

Miura Ori is a noteworthy foldable surface that has been known in origami and rediscovered separately by Miura [1] and [2] Brunner.

It has been used in a fixed roof (Meguro Persimmon Hall, Tokyo) for the acoustic quality of the corrugated surface and it was proposed for a solar antenna [3], where also its kinematic characteristics can be fully exploited.

Geometrically, it constitutes a tessellation of the plane formed by trapeziums all equal to each other, translated along one inclined side and mirrored around one parallel side Figure 1. Kinematically, it achieves a mechanism composed of rigid bodies (the faces) and connected by simple hinges. The mesh is also flat foldable, developable, and can achieve a great efficiency of packaging.

Flat foldability means that in the folded configuration all faces lie in parallel planes, what allows a very compact transversal section. The two conditions ruling locally (for each inner vertex) have been known far back in origami world. With labels referring to Figure 1 these are: (Maekawa and Kawasaki's Theorem) For any inner vertex of the diagram, the sum of alternate position angles $\alpha$ must be: $\Sigma \alpha_{\text {odd }}=\Sigma \alpha_{\text {even }}=180^{\circ}$, and (Maekawa's Theorem) the difference between the number of positive and negative dihedral angles $\rho$ around each inner vertex must be $\left|\rho_{+}-\rho_{-}\right|=2$. [4] Actually, is usually harder to test global flat foldability, but here it is not necessary due to the strong symmetry of the proposed mesh [5].

Moving from origami to engineering, the developability is an important quality: a developable surface can be flattened onto a plane without distortion (compression or stretching) [6]. Developability is achieved when the Gaussian curvature of each inner vertex zero. It is also related to the mesh ability to move rigidly. It is trivial to point out that every strict origami is developable.

A movement is rigid if it occurs just on joints while faces act as rigid members and do not have to absorb the motion strain. Rigidity can be achieved if opposite dihedral angles $\rho$ around each vertex are equal and the same-parity pair are greater than the other pair. To control rigidity, the preliminary relationship between 


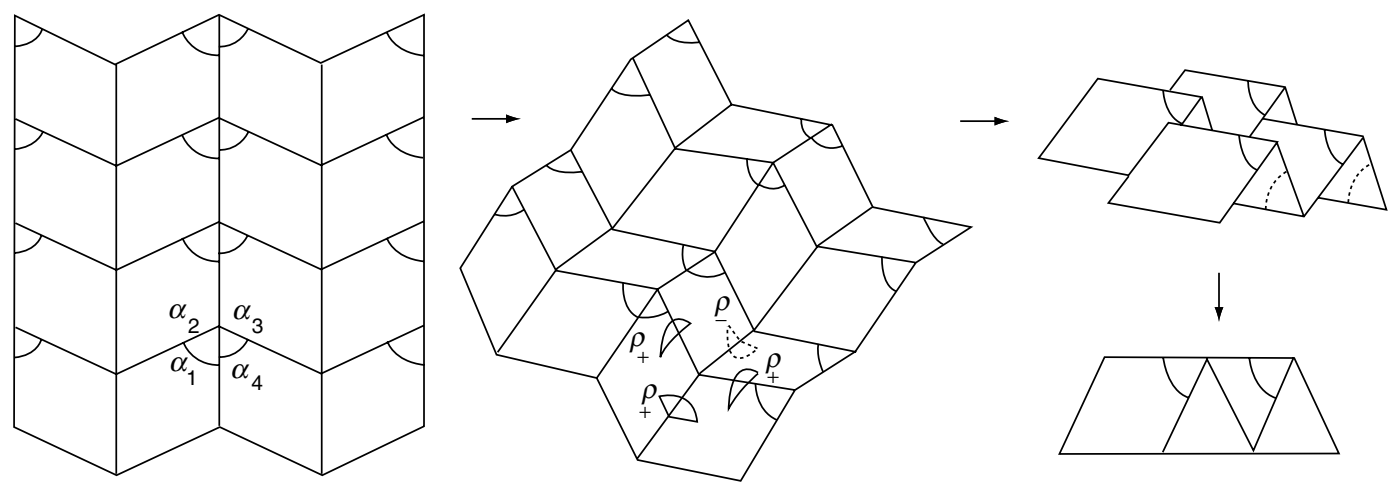

Figure 1. The Miura Ori polyhedral surface at different stages of its movement. The tiling is made by translating the faces in one direction and mirroring them in the other. The opposite dihedral angles (shaded) are equal.

dihedral and position angles has been firstly pointed out in [7] by spherical trigonometry and after it has been proposed in matrix- models in [7-8] and[8-9] in order to generalize the shape of the faces. Tachi has also been combining panels with different orientation and from that he has developed software for the modelling of the surface in real time. In this case, however, the system loses its efficiency of packaging and needs two independent input motions [9].

Working on the classification of flexible assemblies made of four bar linkages, Satchel recognizes the mesh as a special case of the flexible meshes Kokotsakis discovered in 1932 [10], and gives the geometric description of the conditions that ensure the movement.

\subsection{Statement of the problem}

The present work investigates the mobility of the mechanism, that is the number of independent motion parameters which are needed to uniquely describe the position and orientation of its members, i.e., to describe its kinematical configuration. The mobility of the mechanisms is one, which means that just one input variable (here the rotation around one joint) have to be independently controlled to bring the system into a particular position.

The extended Grübler's formula, the basic criterion for counting mobility, states:

$$
M=\lambda(F-J-1)+\sum_{i=1}^{j} f i+h
$$

where:

$M$ : mobility of the mechanism,

$\lambda$ : the number of degrees of freedom of the space in which the mechanism is intended to function;

$F-1$ : number of rigid faces minus the fixed one.

$J$ : the number of joints each with $f i=1$ degrees of freedom; $h$ : number of over constraints.

If the Grübler's equation is used for the minimum configuration of Figure 2, it is:

$$
M=\lambda(F-J-1)+\sum_{i=1}^{j} f i+h
$$

$M=3(4-1-4)+2 \cdot 4+0=1$

If we apply it to the example of Figure 3a, we get:

$M=\lambda(F-J-1)+\sum_{i=1}^{j} f i+h$

$1=3(6-1-7)+7+h$.

$h=0$

And for the example of Figure $\mathbf{3 b}$ it is:

$1=3(9-1-12)+12+h$

$h=1$

Finally, the example of Figure 3c gives:

$1=3(12-1-17)+17+h$

$h=2$

We could go over and we would find that, increasing the size of the rectangular mesh, we need to increase the value of $h$, so to maintain the mobility one.

Where the mobility formula has a positive value of $h$, the corresponding mechanism is said to be overconstrained: its topological structure (the types of
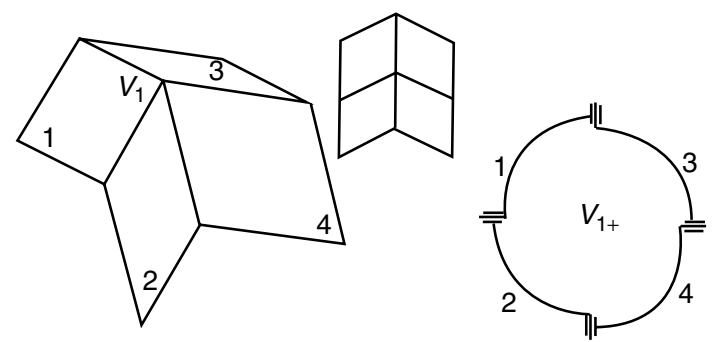

Figure 2. On the left, a polyhedral surface with a Miura Ori pattern; on the right, its spherical linkage mechanism representation. 
(a)

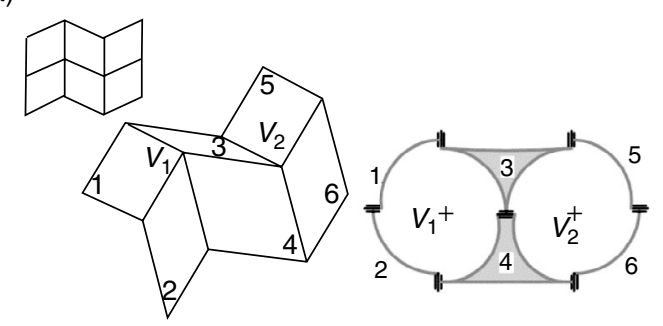

(b)

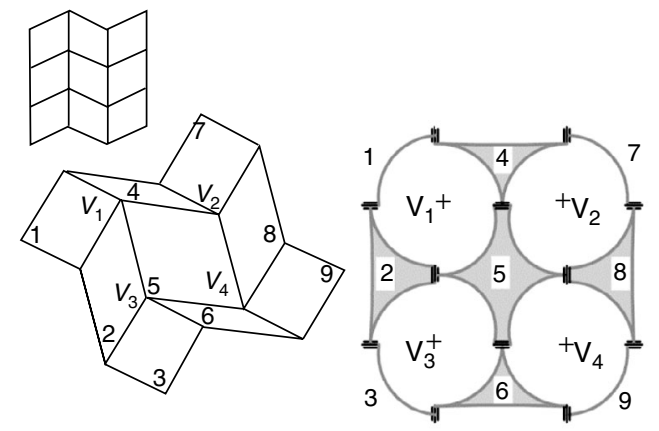

(c)

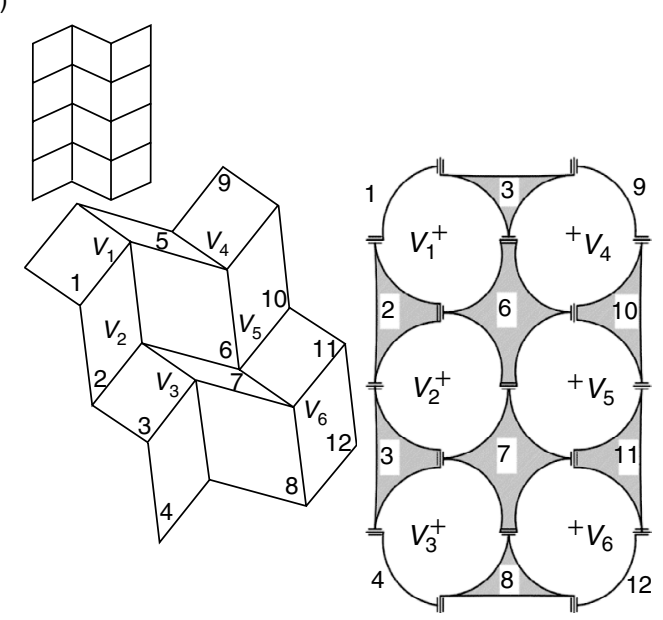

Figure 3. A Miura-Ori pattern and its spherical linkage mechanism representation. (a) 6 faces generate a mechanism made of two 4-bar linkages constrained by each other. (b) 9 faces generate an over constrained mechanism made of four 4-bar linkages arranged in a loop. (c) 12 faces generate more 4-bar linkages and increases the number of loops and so the degree of over constraint.

joints and relationships among them) indicates that it is a structure, while its geometry (the shape of the rigid bodies and the relationship between joints' axes) allows it to behave as a mechanism.

Overconstrained mechanisms, once they are fixed, become hyper static structures and so are especially suitable in those applications where stiffness and stability to loads are important. On the other hand, since these mechanisms have more constraint parameters than what the joints types and number would need, it is also possible to remove excessive members and still to obtain the same movement, so constructing a lighter, customized and cheaper system. The latter propriety will be exploited in the present paper, in order to develop kinetic polyhedral surfaces, which overall shape can be customized for practical or aesthetical reasons.

Firstly, we need to formulate a simple and effective description of the mobility of the mechanism that does not rely on the overall shape of the surface.

Mobility had been previously outlined in [11]. Here, the mechanism is analysed under a static point of view, recognized as, externally, determinate and internally overconstrained. Mobility is then outlined referring to the number of static unknowns. The more members compose the surface, the more becomes difficult counting the degree of overconstraint, due to the presence of parallel and intersecting axes, nested loops, and in general geometrical conditions. The reference in particular takes care of the presence of loops by modelling some revolute pairs as spherical joints and contact points case by case, in a recursive way, which does not end to a straightforward approach.

A general approach is presented in [12]. The mechanism is modelled as a pin-jointed bar framework, where each face is substituted by five bars (one diagonal bar for planarity and four at the boundary), while each original vertex is replaced by a revolute joint with three independent parameters.

It results: $M=b-3 V-h$ where $M$ is the mobility; $b$ the number of bars; $V$ the number of vertexes and $h$ the number of over constraints.

Despite the above equation gives the right results, it needs the transformation of the panel system into a bar mechanism, adding virtual elements and calculations.

In order to gather the geometrical features, here the mechanism of the polyhedral surface is directly investigated.

\subsection{Spherical linkage analogy}

In Figure 2, four faces reciprocally connected are represented in a generic folded state. The axes of rotation of the revolute pairs, of the hinges, are aligned with the boundary of faces and intersect in a common point $V_{1}$. Moreover, since the faces are considered rigid bodies (1, 2, 3, 4 in figure), then the motion is rigid. As showed in [14], the movement of such a polyhedral surface can be directly described through a spherical linkage. The analogy holds of course until the extreme positions of the mechanism are discarded. 
When the mesh is completely unfolded or completely folded, in fact, all the joints would be on the same plane or coplanar.

Under the above restriction, vertex $V_{1}$ will be the centre of the sphere, while the joints are the vectors connecting it to the spherical members $1,2,3,4$. The result is a four bar linkage with revolute pairs only, and, as every linkage of this type, it has mobility one [14].

\section{MOBILITY}

\subsection{Two-strips condition}

The spherical linkage analogy allows us to express the following:

\section{THEOREM}

If the arrangement of faces generates a double-line path, then the Miura Ori surface is a mechanism with mobility one.

\section{PROOF}

We consider bigger and different surfaces. If there are five faces, clearly mobility of the mesh increases to two. Instead, a sixth face causes some ambiguities: if the face is connected to the previous through just one joints, then mobility increase. Instead, if the face shares two joints with the priors, so that all together they create a double-strip (Figure 3a,) another spherical linkage is created. For construction, all the members have equal length. Focus now on the added spherical linkage: it shares two members $(3,4$ in figure) with the priors. Every four bar linkages, spherical or planar, made of revolute joints with two rigid members in common are constrained to move together. Thus, here also the movement of one linkage uniquely determines the movement of the other.

In Figure 3b we have a square surface. Again rotating one joint all the faces move accordingly. In the spherical representation, each four-bar linkage shares two members with both the linkages adjacent to it.

If we would write the compatibility equations, i.e., the equations geometrically relating the input and output variables of a mechanism, we will end having two linearly dependent equations describing one of the members $1,3,7$ or 9. In fact, once decided the input angle, for example the angle between members 1 and 2 , we would be able to describe the position of members 4 and 5 also, closing the linkage centred in $V_{1}$. Then, we could move counterclockwise and use the position of members 2, 5 to describe the linkage centred in $V_{3}$. Then we could use members 5,6 to describe the linkage centred in $V_{4}$. At this point, we would know the position of all the members except $n$. 7. This variable could be described both through the linkage centred in $V_{4}$ and the one centred in $V_{1}$. Member 7 is excessive: we do not need it to find out all the rotation angles and it could be removed without consequences for the overall movement of the mechanism. If we proceeded starting from another linkage, and/or moving clockwise, we would have find one different excessive member, nevertheless, it would had been just one, that is member 3,7 or 9. The same holds in the polyhedral surface (Figure $\mathbf{3 b}$, on the left): we can remove face $1,3,7$ or 9 and still the surface will fold the same way. Especially, we point out that the polyhedral surface after removing whatever one excessive member would be made of faces each doubly connected with the others, forming a double line strip.

In Figure 3c we have 12 faces corresponding to 6 spherical linkages. We could start the kinematic analysis from the linkage centred in $V_{1}$, and then proceed counter clockwise to the linkages centred in $V_{2} . V_{3}, V_{6}$. At this point, we know the position of all the members except n. 9 and n. 10. We could calculate them using the information from the linkage centred in $V_{1}$ or the one centred in $V_{5}$. And we could remove members 9 and 10 without affecting the movement of the mechanism. Again, we could start the analysis from another 4-bars linkage, move in a different way, and always we would have needed to know the position of two members from one linkage to describe the following. As before, in the corresponding polyhedral surface we could remove the found excessive faces and we would still have a double-strip of faces.

The result is useful under the design point of view. We can variously remove faces from a compact shape, and obtain a great range of shapes: we still preserve the required mobility, until the double-line condition hold, as in the samples of Figure 4.

\subsection{Mobility formula}

Recalling the result in (1.1), we can identify the number of overconstraints $h$ with the number of excessive members in the spherical linkage analogy and with the corresponding faces in the polyhedral surface. We call thus Excessive Faces, $F_{e x}$, the maximum number of faces that can be removed without affecting the mobility of the mechanism.

Then the Grübler's formula can be rewritten as:

$$
M=\lambda(F-J-1)+\sum_{i=1}^{j} f i+F_{e x}
$$

Actually, the Grübler's equation is usually adopted for counting the mobility of one spherical linkage, 


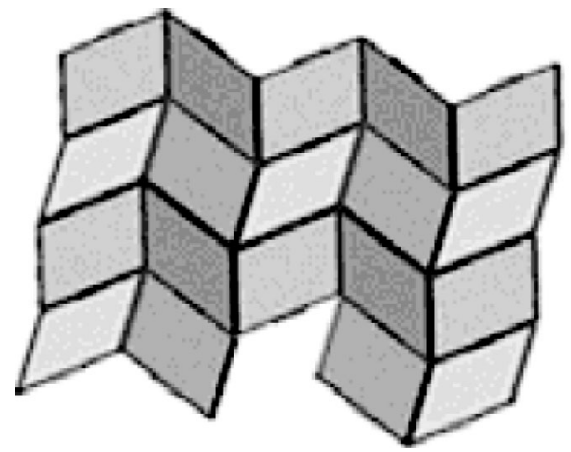

(a) $M=1$

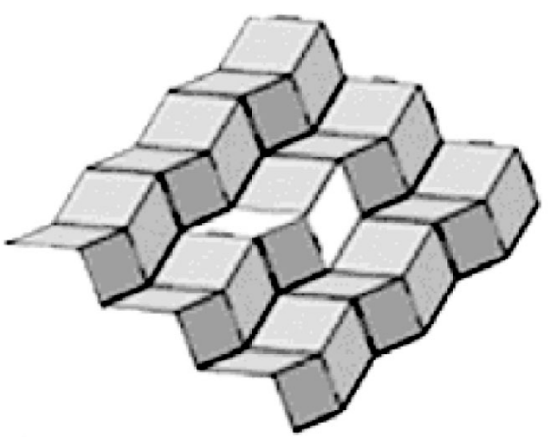

(c) $M=1$

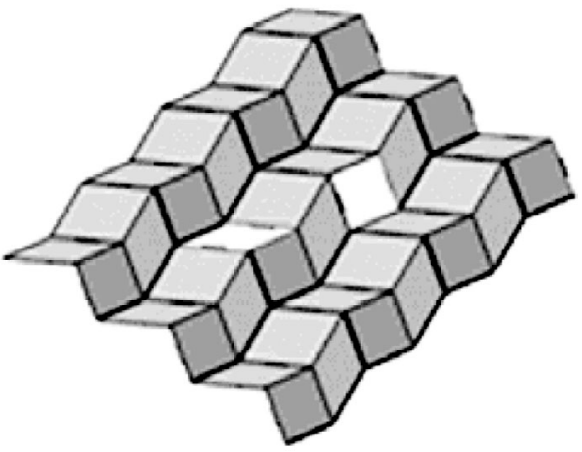

(b) $M=1$

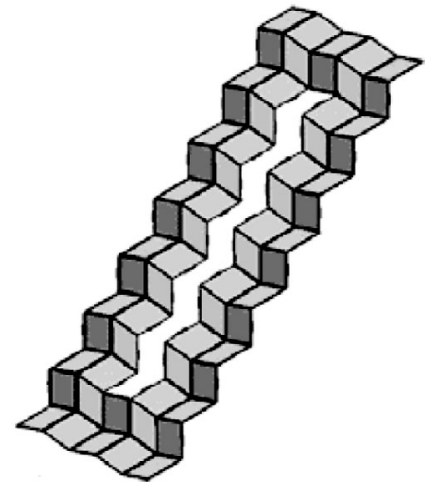

(d) $M=1$

Figure 4. Until there is a continuous double line path involving all the remaining faces of the mesh, faces can be removed without affecting mobility. The samples have all mobility one.

centred on one sphere. Here we have more linkages but we can still apply the formula because the two spheres can be made coincident due to their construction. Consider the example in Figure 3b. The loop of faces 1, 2, 3, 4 shares two faces with loop 3.4.5.6 and, from the description of the surface in $\S 1.1$, faces 5, 6 and 1, 2 are the mirrors of faces 3, 4 along the common sides. Similarly, the two linkages 1, 2, 3, 4 and 3, 4, 5, 6 have members 3, 4 in common and the others 1,2 and 5, 6 are both mirrors of 3, 4. We can imagine therefore to mirror back one linkage above the other, and we get now two coincident spherical linkages, centred on the same sphere and connected to each other.

\subsection{Number and position of the excessive faces}

Let's apply the mobility formula to the example in Figure 5a.

$$
\begin{aligned}
& \quad M=\lambda(F-J-1)+\sum_{i=1}^{j} f i+F_{e x} \\
& 1=3(170-313-1)+313+F_{e x} \\
& F_{e x}=120
\end{aligned}
$$

Looking to Figure $\mathbf{5 b}$, we can remove the excessive faces, proceeding freely in various ways and, since we respect the double-strip condition, the mobility will stay unchanged. Nevertheless, the maximum number of removable faces, Fex, can be obtained only if the removed faces are grouped together in no more than two assemblies (Figure 5c).

Finally, looking to Figure 5d, we also note that the number of Fex can be directly inferred by the overall rectangular dimension of the surface. In fact, whatever the final shape of the system, for the case in which $M=1$ the removable faces are equal to $\left(L_{1}-2\right)\left(L_{2}-2\right)$, where $L_{1}, L_{2}$ are the number of faces in the overall boundary length dimension of the discrete conjugate net.

In the represented case, it is:

$$
\begin{aligned}
& L_{1}=10, L_{2}=17 \\
& F_{e x}=\left(L_{1}-2\right)\left(L_{2}-2\right)=120
\end{aligned}
$$

Of course, if we wish to design a mechanism with mobility 2 , the maximum number of faces will be one more of the previous. We can thus write:

$$
F_{e x}=\left(L_{1}-2\right)\left(L_{2}-2\right)+(\mathrm{M}-1)
$$

\subsection{Two-strips condition}


(a)

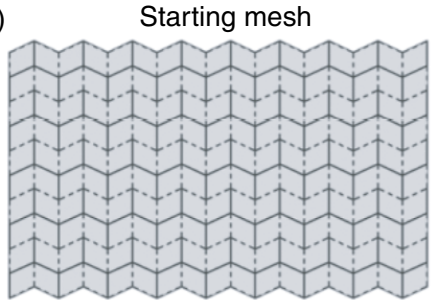

(c)

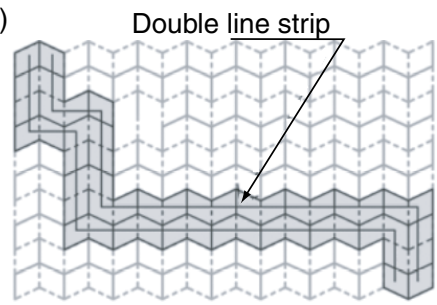

(b)

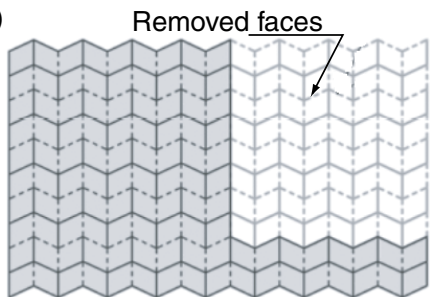

(d)

Maximum number

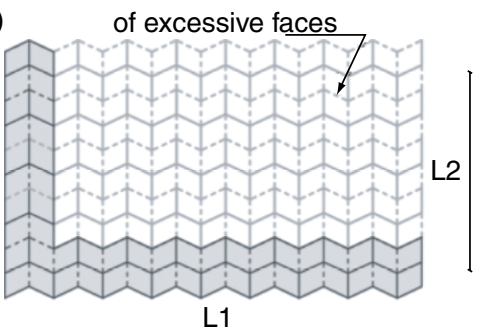

Figure 5. Starting from a rectangular surface (a), it is possible to remove faces until a two-parallel lines path is preserved (b, c). Desired the wished mobility, the number of excessive faces can be easily calculated from the overall dimension of the initial surface (d).

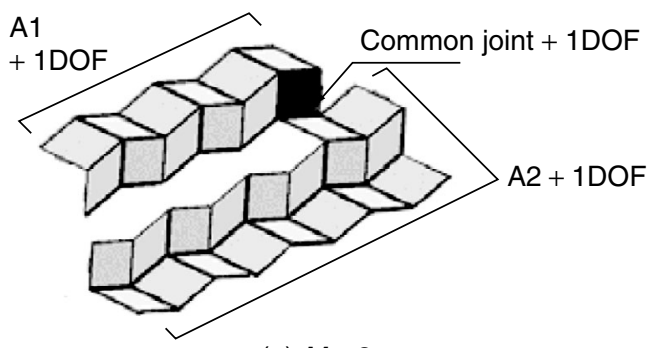

(a) $M=3$

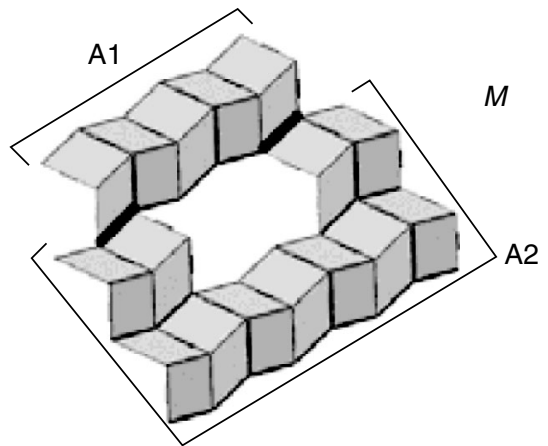

(c) $M=1$

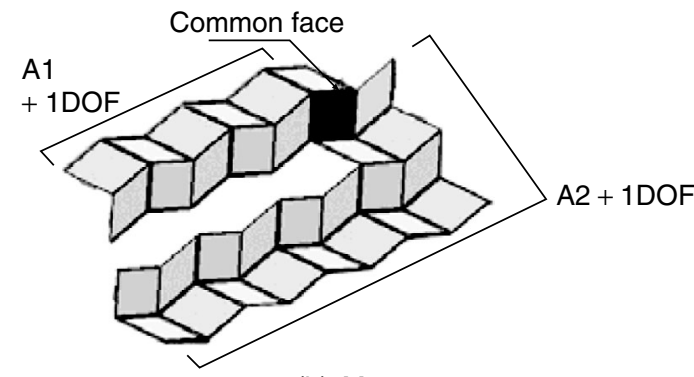

(b) $M=2$

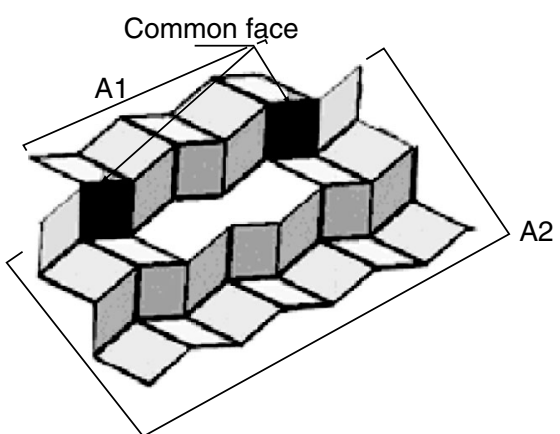

(d) $M=1$

\section{An: Area made of double line strips \\ : Faces common to two areas \\ $:$ Joints common to two areas}

Figure 6. (a) If double-line areas share one joint thus 2 independent variables are added; (b) if they share one face, still the two areas can fold independently; (c) if they share at least two joints, whatever is their distance, they generate a mesh with mobility one; (d) if they share two faces, mobility is one.

It should be observed that, to maintain mobility unchanged, the double line condition presented in $\S 2.2$ does not impose that the strips of faces are adjacent to each other. In Figure 6, in case a) two double-strips meshes share just one joint. Each of them folds independently and another variable is due to the rotation of the common joint: the total mobility is three. In case $b$ ) they share one face, they have two joints in common, and the folding of one area sets just the inclination of the second area with respect to the common face. The overall mobility is two. In case $c$ ) they share two joints. The position taken by the 


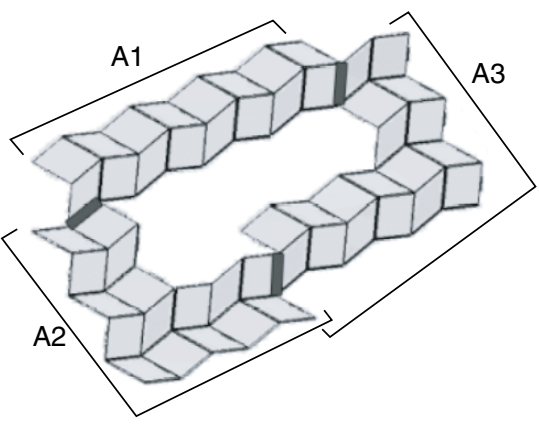

(a) $M=1$

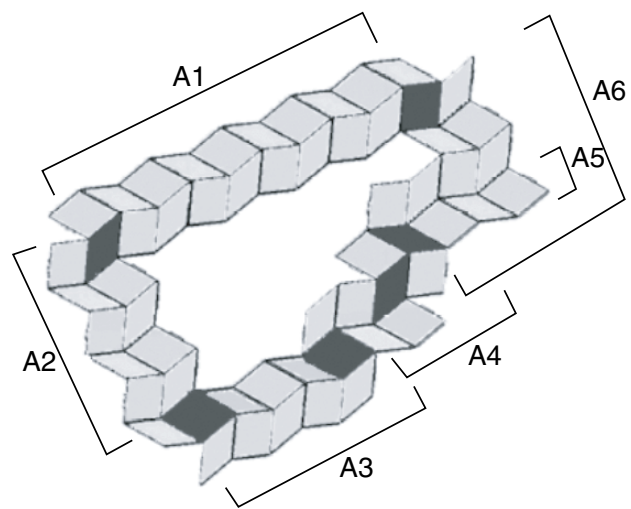

(c) $M=1$

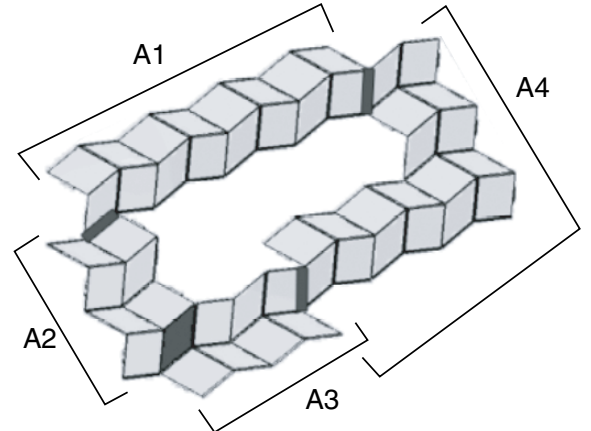

(b) $M=2$

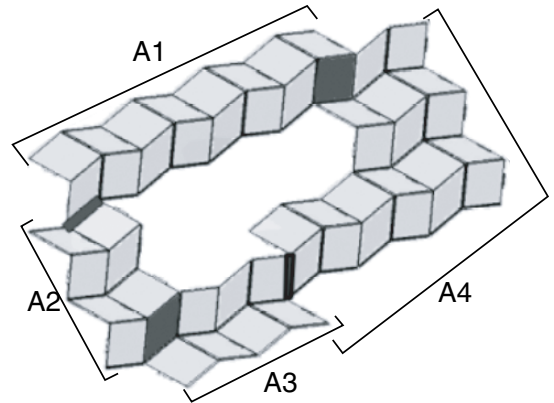

(d) $M=1$

An: Area made of double line strips
: Joints common to two areas
$/$ : Faces common to two areas

Figure 7. Survey of loops made of double-strips areas. (a) Loop of three areas connected by joints. (b) Loops of more than three areas with three consecutive one-joint connections. (c) Loop made of areas connected by faces. (d) Loop of more than three areas without two consecutive one-joint connections.

common joints during the movement of one area constrains the other area to fold accordingly. The overall mobility is one. In $d$ ) they share four joints and from above mobility is one. Hence, the double line condition does not mean strictly that faces have to be arranged in a large strip: they have just to create two parallel strips, whatever is their distance.

Let's now extend the procedure to the cases where more double-strips areas are involved. If they generate an open tree, then

Consider now a closed loop made of three doublestrips areas. If the three areas are connected to each other each by one joint, the movement of one area imposes the distance between the other two, in fact they forms a triangle of areas. Hence the mobility does not increase (Figure 7a). Even more so, if each of the three shares with the adjacent one face, then mobility does not increase.

If the involved areas are more than three, connected each other by one joint, when one moves it cannot univocally set the position of the others, and mobility increases (Figure 7b).
The use of common faces avoids the problem, since when one moves, it constraint the rotation of the adjacent areas (Figure 7c).

We can still use one joint connections in a closed loop made of more than three areas, but connections made of just one joint have not to be adjacent. Figure 7d illustrates this case: there are two connections made of one joint, but these are separated by other connections made of one face.

We can summarize the previous survey in the following note.

\section{REMARK}

Two or more double-strips areas $A$ can be connected to each other with mobility one in one or more closed loops if each two adjacent areas are connected by at least one joint or one face, so that there is not a loop of more than three areas respectively connected to each other by just one joint. (2.5)

\section{CUSTOMIZATION}

\subsection{General rule}

The high redundancy of the mechanism allows a high freedom in design. We already discussed the possibility 


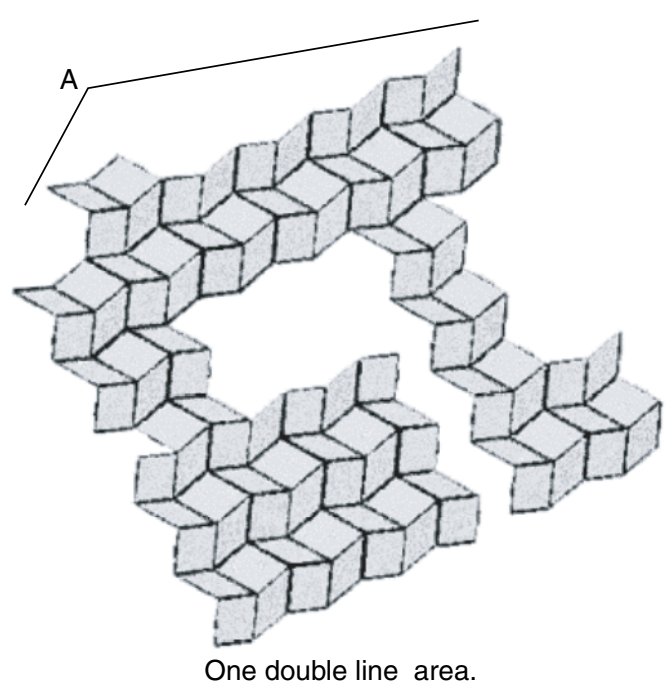

(a) $M=1$

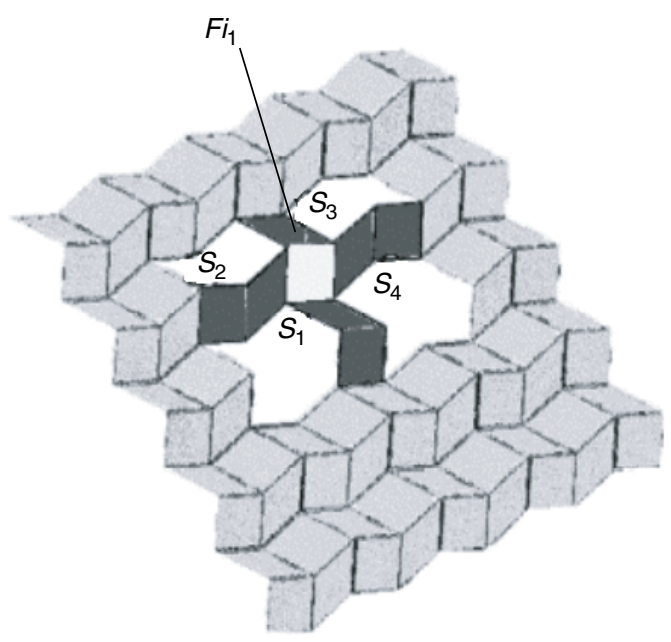

Single line strips, $S i_{1, \ldots, \mathrm{n}}$. one intersection face. Fi.

(c) $M=1$

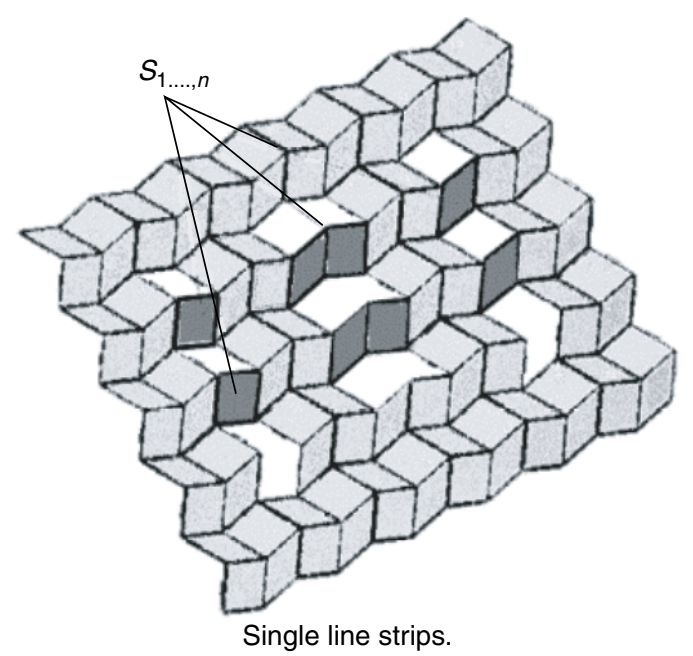

(b) $M=1$

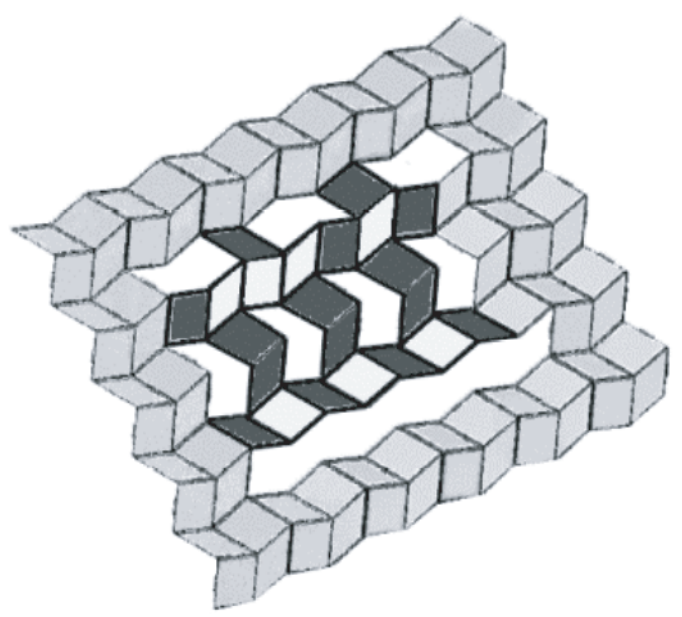

Loops of single line strips, $S i_{1, \ldots, n}$, and intersection faces, $F i, \ldots$, m.

(d) $M=1$

Figure 8. The arrangements of the double -line area and of single-line strips as well allow customizing the shape of the polyhedral surface.

to remove all the excessive faces by using the doublestrips condition. We can thus customize the shape of the double-strips area $A$ (Figure 8a). Then, if our intent is not to remove all the excessive faces, but to obtain a special arrangement, under some geometrical conditions we can except the double-strip rule. These can help to customize the system for special needs. For example, it is needed to construct a foldable roof, and it should be partially panelized; or there is a mechanism that has to cover a specific path, discarding the panels whose motion is not of interest or unwanted.

To investigate these possibilities, we define singleline strip, $S n$, a straight strip of $n$ faces where each face is connected to no more than other two faces, in opposite sides (Figure 8b); we call strip's faces, $F s$, those that belong to such single-line strips. A single- line strip can be connected at its extremities to a double-strips area $A$, left free, or connected to an Intersection Face, $F i$. We define an Intersection Face, $F i$, the one face that separate two or more single-line strips (Figure 8c).

In particular, given a double-line area and singleline strips in between, the single-line strips do not increase the mobility of the mechanism if they abide by the following necessary and sufficient condition:

\section{REMARK}

Every single-line strip, $S$, has to be composed of no more than two faces, $F s$, and be connected at its ends to a double line area, or be inserted in a loop of such single-line strips together connected at least twice to the double-line area $A$. 
If Intersection Faces, $F i$, are present: each connects at least two strips so that all together $(F s+F i)$ they are strictly fewer than five faces; there are not four strips each made of two faces that are pairwise collinear. (3.1)

The demonstration of the above statements is developed in the following paragraphs.

\subsection{Single-line strips}

If a single-line strip is connected at both its extremities to a double-strips area, and is composed just by one or two faces, then it cannot rotate independently from the rest of the mesh. Looking to Figure 9, the side $l$ of the single-strip faces and their reciprocal distance $2 d_{1}$ generate a triangle. The dimension of $l$ is due by construction; $d_{1}$ is given by the adjacent faces. Then, in every phase of movement, discarded passing though singularity position, the rotation angles $\alpha_{n}$ is the same for all the faces and is the only variable to be determined (the mobility of the mesh).

In Figure 10 the single-line strip is again connected at its extremities to a double-line area, but it is composed of four faces. The dimension of $l$ is due by construction; $d_{3}$ is given by the faces behind. For each value $\alpha_{1}$ of the rest of the mesh, it is necessary to fix one more angle $\alpha\left(\alpha_{2}, \ldots, \alpha_{5}\right.$ in figure $)$ to calculate all the variables. The more faces compose the single-line strip, the more variables stay independent from the main mesh. The mobility of a mesh where there are one or more single-line strips can then be calculated as follow:
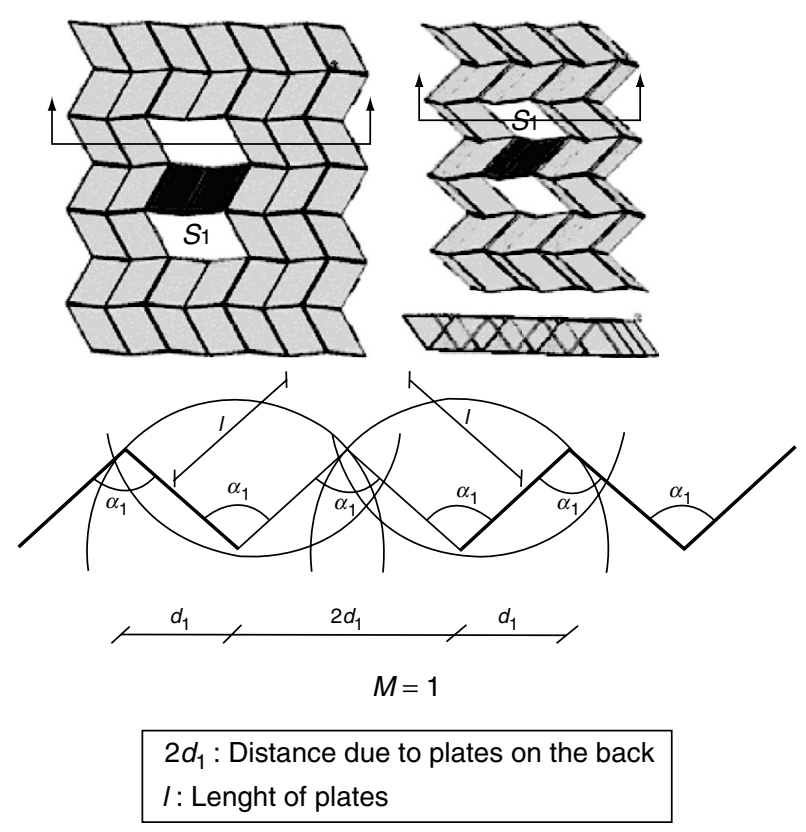

Figure 9. If a single-line strip is connected at both its extremities to two -lines areas and is composed by just one or two faces, then it is constrained to move accordingly to the rest of the mesh.
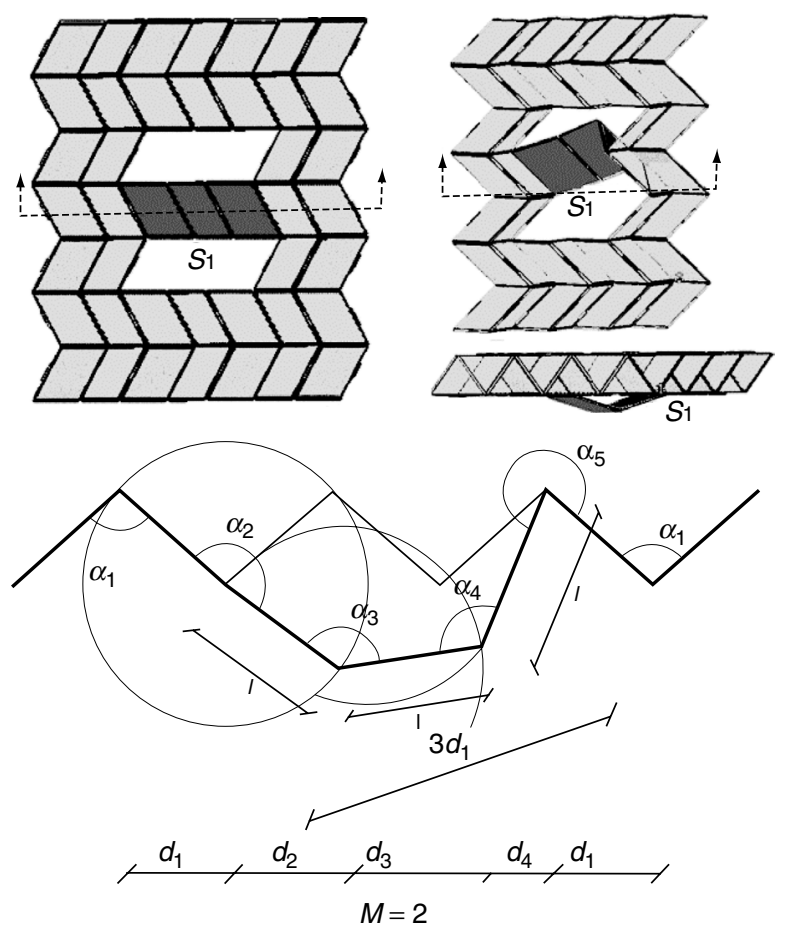

$2 d_{1}$ : Distance due to plates on the back
l: Lenght of plates

Figure 10. If a single-line strip longer than two faces is connected twice to a double-line area, it can rotate independently from the main mesh, so mobility increases.

$$
M=M_{A}+F_{S}-S^{1}-2 S^{2}
$$

where $M$ is mobility, $M_{A}$ stays for the mobility of the double-strip area, $F s$ is the number of faces that compose all the single-line strips together; $S^{1}$ is the number of single-line strips made of just one face, $S^{2}$ is the number of single-line strips made of just two faces.

The deployable mesh in Figure $\mathbf{8 b}$ has mobility one and exhibits a grid of horizontal and vertical voids, separated by single-line strips.

\subsection{Angle made by single-line strips}

If two single-line strips are connected to the same intersection face $F i$, so that all together $(F s+F i)$ they are strictly fewer than five faces, they constrain the each other movement and therefore mobility does not increase. Looking to Figure 11, two strips intersect on the middle of the mesh. The angle $\alpha_{1}$ and distance $d_{1}$ are given by the overall movement of the mesh. The position of the intersection face $F i$ is constrained in the $x$-direction by the single-line strips in behind and in the $y$-direction by the adjacent strip. Then, the longitudinal overall dimension of each single-line strip is constrained and the faces completely follow the movement of the overall mesh. The same holds if both 

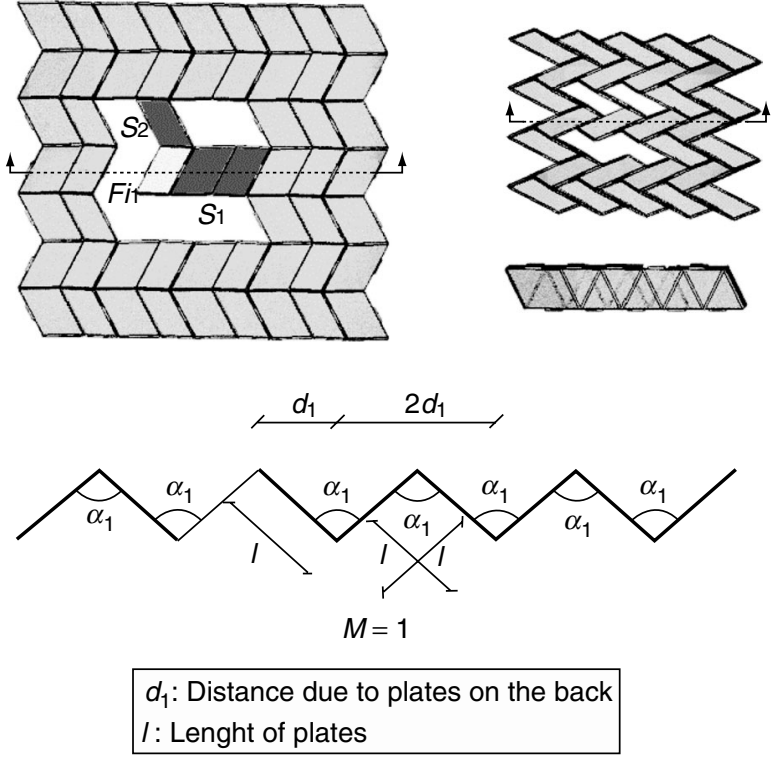

Figure 11. If single-line strips generate an angle, and the intersecting face plus the number of their faces is less or exact four, then their rotation is constrained.

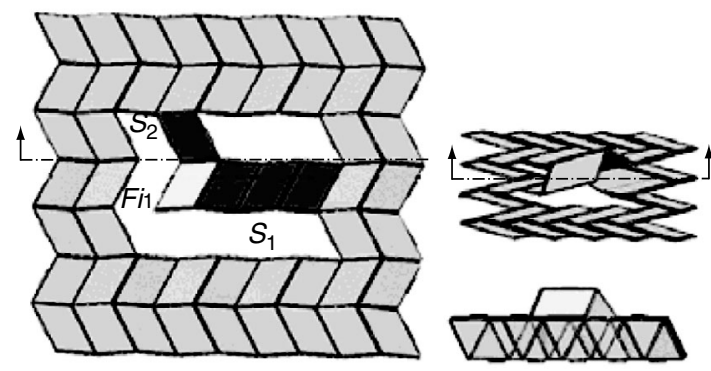

$3 d_{1}$

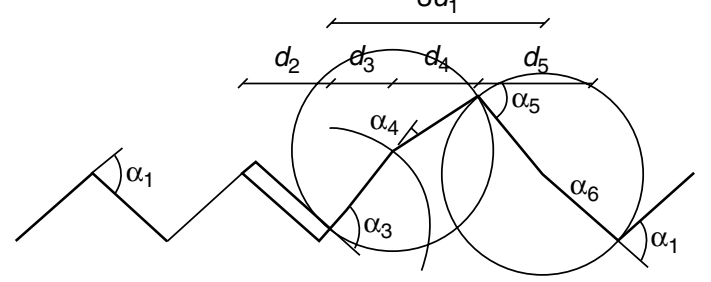

$d_{2}$. Distance due to plate on the back

$3 d_{1}$. Distance due to plates on the back

$$
M=2
$$

Figure 12. If single-line strips generate an angle and the number of their faces, excepting the intersection face, is equal or more than four, mobility changes accordingly.

strips are composed of just one face. Instead, mobility increases if the strips, including the intersection face $F i$ , are globally made of five or more faces, as illustrated in Figure 12. Thus:

$$
\begin{array}{r}
M=1+F i+F s-4 \text {, with } F s \geq 4 \\
M=1, \text { with } F s<4
\end{array}
$$

where $F i$ is the intersection face.

A system with mobility one that takes advantage of this possibility is shown in Figure 8c.

\subsection{Intersection of single-line strips}

The observations done regarding single-line strips and their matching can be further developed in case where single- line strips are connected to the same intersection face, creating $\mathrm{T}$-like or $\mathrm{X}$ like intersections.

If three single- line strips intersect, and their length is short enough, that is, they are made of just one or two faces, then their combination does not affect mobility.

Looking to Figure 13, each strip is composed just of one or two faces. Calling $S_{1}, S_{2}, \ldots, S_{n}$, each singleline strip, then $S_{1}$ and $S_{2}$ constraint each other, and the same is for $S_{6}$ and $S_{7}$. So, at each step of movement, the positions of the intersecting faces $F_{i 1}, F_{i 3}$ are given. In the middle of the mesh, $S_{3}$ is connected on the left to the constrained $F_{i 1}$ and creates an angle of less than 5 faces with $S_{4}$ and $\mathrm{F}_{\mathrm{i} 2}$. The rotation angles of $S_{3}, S_{4}$ and $F_{i 2}$ therefore are also constrained. Finally, $S_{5}$ is connected on the right to $F_{i 3}$, which rotation is given, and on the left is connected to $F_{i 2}$, which rotation is also given. Thus, $S_{5}$ have to move accordingly to the rest of the mesh. Hence:

\section{REMARK}

The strips made of one or two faces, combined in one or more T-like intersections, do not affect the mobility of the system until they are globally connected twice to a double -line area.

A special condition occurs if four strips each made of two faces are pairwise collinear. Looking to Figure 14, $S_{1}, S_{2}$ meet in $F_{i 1}$ and are connected to the same double
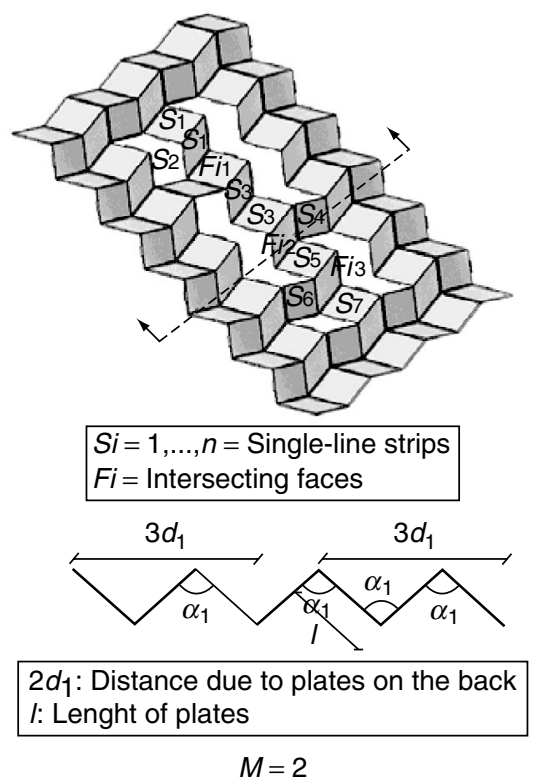

Figure 13. Single-line strips each made of one or two faces can create loops connected to the double-line area, without affecting mobility. 

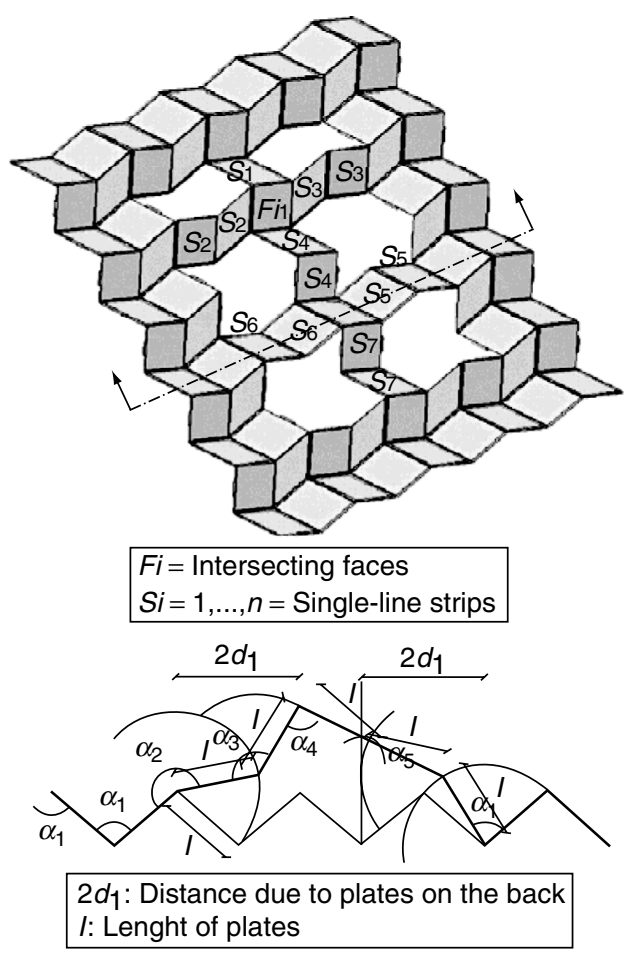

$M=2$

Figure 14. If in a loops of single -line strips there are four strips pairwise collinear, they add an independent rotation parameter.

-line area and so constraint each other. The same holds for $S_{1}$ and $S_{3}$.

Below, $S_{6}$ and $S_{7}$ meet at an angle in $F_{i 2}$, and are totally composed of four faces. For what demonstrated in $\$ 3.3$, they add one independent variable to the overall mechanism: we need to decide the rotation angle between $F_{i 2}$ and $S_{6}$ or between $F_{i 2}$ and $S_{7}$ so to arrest the free rotation of $F_{i 2}$. On the right, the same relationship holds between $S_{7}, S_{5}$ and $F_{i 2}$. Since we just defined the orientation of $F_{i 2}$, this occurrence here does not increase mobility. Finally, $S_{4}$ is a single-line strip composed of two faces. For what demonstrated in $\S 3.2$, it does not increase mobility. Together, the mechanism has mobility two, one due to the overall movement, one due to the orientation of $F_{i 2}$. This special circumstance happens wherever four singlestrips, each made of two faces, are in pairs collinear. It is possible to state that:

\section{REMARK}

Single- line strips can be connected together without affecting mobility if each strip is composed by one or two faces, and there are not four strips each made of two faces that are pairwise collinear.

\subsection{Grids of reconnecting strips}

In $\S 3.4$ it has been shown that single -line strips can be connected to other single-line strips without changing

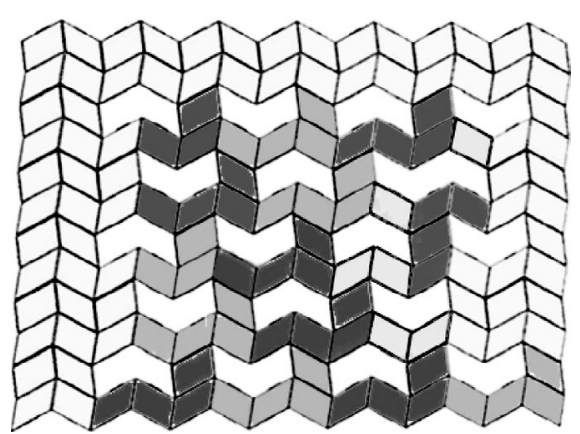

(a) $M=1$

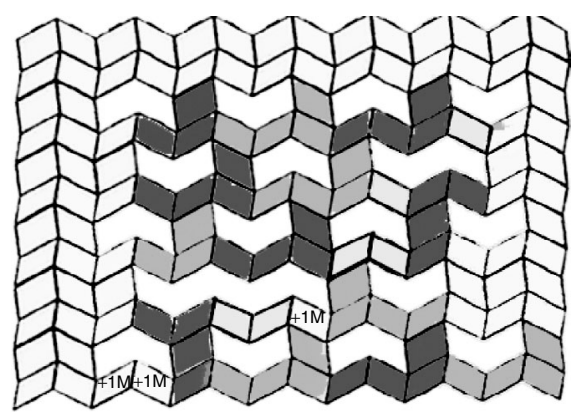

(b) $M=4$

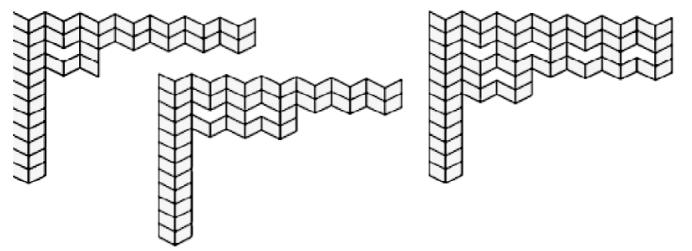

(c) Design process of the mesh in figure a

Figure 15. In case (a) starting from the double line area (light grey), a first group of single -line strips and intersection faces are constrained by the double line area. A second group is connected to it and to the double line area. So on, each following group is constrained twice by the previous groups and / or by the double line area. The overall mobility is one.

(b) There is one face missing respect to the previous group, which affects mobility of more strips constructed upon it. The total mobility is 4 . (c) Connecting process of the singleline strips.

mobility. The necessary condition, in fact, is that the strips composed by no more than two faces belong to a loop connected to a double line area. Going further, strips can also generate multi-loops of this kind. The sample of Figure 15a shows a sequence of horizontal and vertical strips connected each other, and its mobility is one. Figure 15b, instead, shows a mesh with just one more face removed. Its mobility is four, since the removed face breaks more loops of single -line strips. The design process of this kind of surfaces is showed in Figure 15c: once decided the shape of the double line area, a first sequence of single-line strips and intersection faces is added, constrained by the main mesh. This way, it actually acts like the double-strip 
area, and can be used to constrain other single-line strips constructed upon it. It is possible to state that: REMARK

Starting from a constrained area, valid (short enough) single-line strips and their intersections can be connected each other in sequences and constraint each other.

\subsection{Connection of double-line areas}

If single -line strips connect separated double-line areas, generally the only constraint to the rotation of the strips is due to the distance between the two double-line areas. The shorter single-line strip with its rotation sets this distance; all the other single-line strips involved, to stay connected to the double-strips areas, have to fold accordingly (Figure 16a).

In case where the joints connecting the strips to a double-line area are collinear and strips are composed of just one or two faces, like in Figure $\mathbf{1 6 b}$, then rotation angles $\alpha_{2}$ are constrained to be all the same. In fact, since the strips are made of the same number of equal faces, they can cover the distance $d_{2}$ between double-line areas all by rotating the same amount. Moving further, if two of such strips are connected to the same double -line area with non-parallel joints, then the rotation angle of the connecting joint is constrained to rotate accordingly also to the rest of the mesh, otherwise faces would

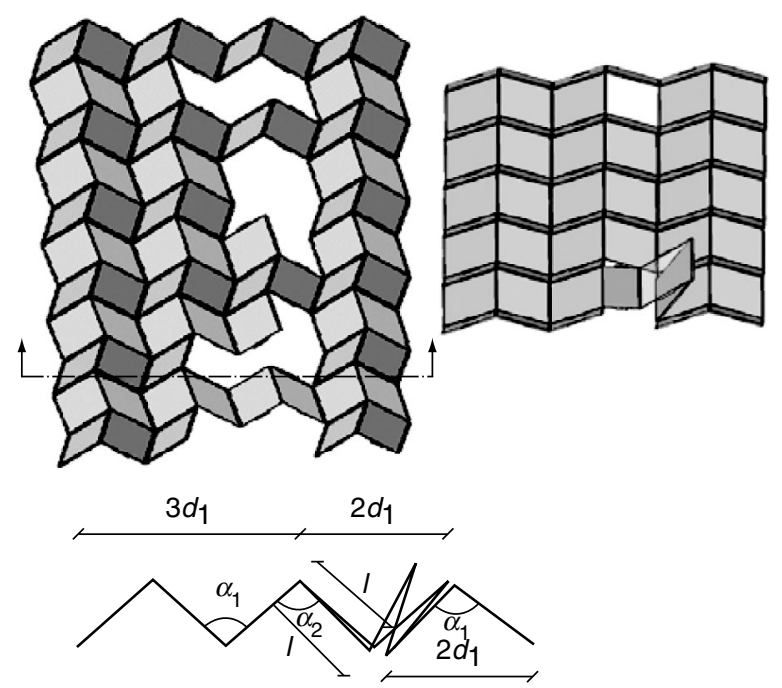

(a) $M=3$

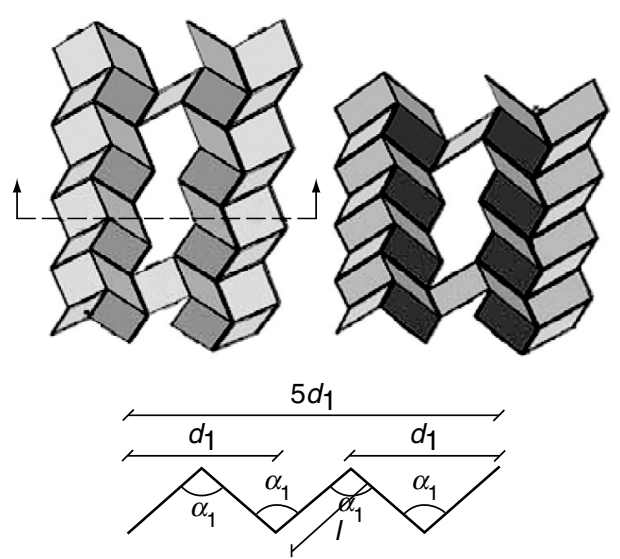

(c) $M=1$

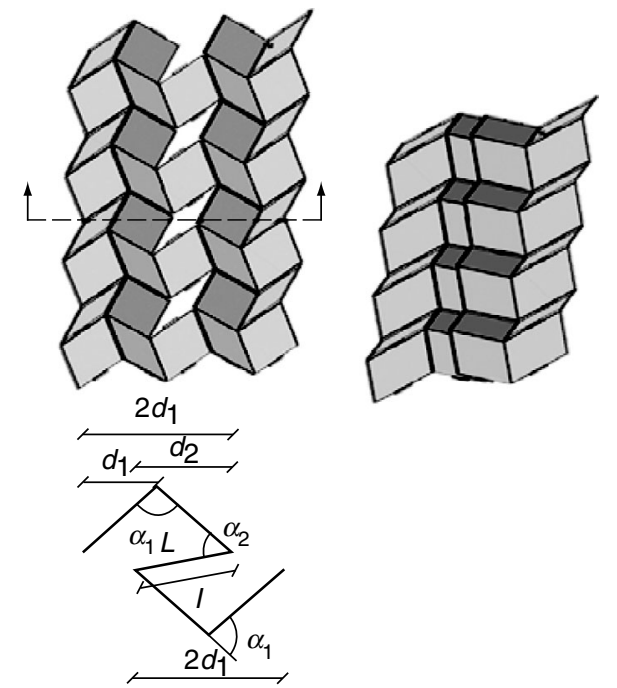

(b) $M=2$

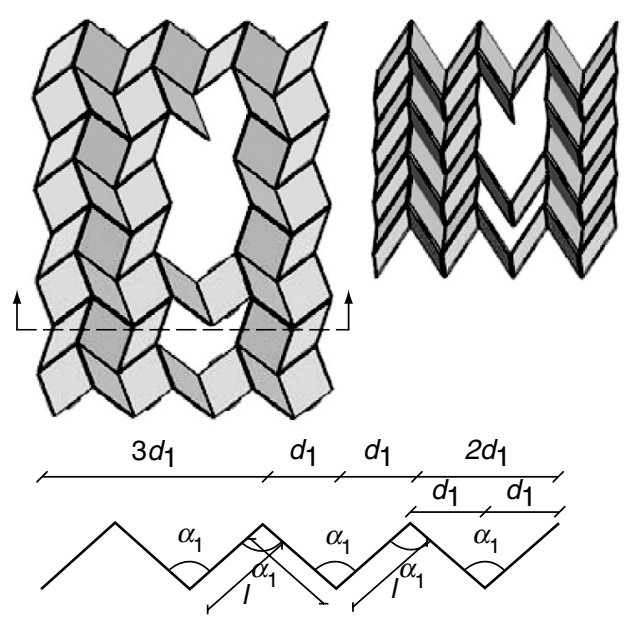

(d) $M=1$

Figure 16. (a) If single -line strips connect separated double-line areas, the distance between the two double-line areas change linear along adjacent strips, but this requirement can be differently satisfied, depending on the number of connecting strips and their faces. (b) If the strips are made of a single face, and their joint are collinear, then their rotation angles have to be equal to each other. (c) If the faces' joints are not collinear, they rotate accordingly to the double-lines areas and mobility is one.

(d) Mobility is one until there is at least one strip made of one face, and the others no more than two and at least two joints connecting strips and double-line areas are not collinear. 
intersect each other (Figure 16c). The same holds until at least one strip is made of just one face or one joint (strip of zero length), the others of no more than two faces (Figure 16d). Thus:

\section{REMARK}

Two double line areas are constrained to fold together if they are connected through one strip made of one face or one joint and at least another strip, composed of no more than two faces, so that the gap between at least two strips is an even number of faces.

We can write:

$M=+F_{6}-2 S+S^{1}-F i+q$

where:

$M$ is mobility,

$F s$ : the number of faces that compose all the singleline strips together;

$S:$ the overall number of single-line strips,

$S^{1}$ : is the number of single-line strips made of just one face,

$q: 0$ if the gap between at least two strips is an even number of faces.

$q: 1$ if the gap between every two strips is an odd number of faces.

\section{GRIDS OF RECONNECTING DOUBLE-LINE AREAS}

The information gave so far should allow the design of custom Miura Ori meshes with mobility one. To design them, there is no need of software since the constraints are based on simple rules and basic calculation. Moreover, the overall sketch could be made also without rules, because dimensions are expressed as number of equal faces.

One could design a simple double strip path, as in Figure 15, or could design a more complex shape, with empty areas. We would like to describe the design process for such a surface, exemplified in Figure 18.

Firstly, we made a sketch of the final model (Figure 17a). Then we began to remove the first faces, which separated areas $A_{1}$ and $A_{8}$ from the rest of the mesh. From Remark 3.7 all the areas fold accordingly. In fact, between $A_{1}$ and the central mesh there are two single-line strips with an even number of faces in between. Both strips have 0 length (one joint per strip). The same holds between $A_{8}$ and the central mesh. (Figure 17b)

Then, we needed to divide the main area into two parts $\left(A_{2} A_{6}\right.$ and $\left.A_{3} A_{7}\right)$ to host the central void. Let's focus on one of these parts and on its connections. From $\S 2.4, A_{2} A_{6}$ and $A_{1}$ are two double-strips areas connected by a joint: each of them moves with its own mobility, and another degree of freedom is due to the joint connecting them. The same holds between $A_{2} A_{6}$ and $A_{8}$. (a)
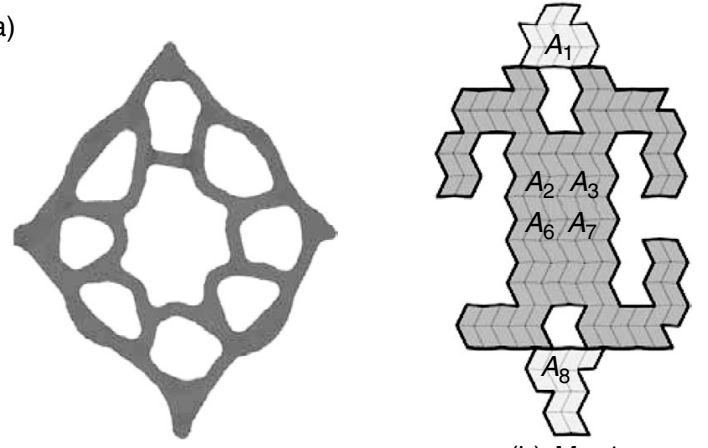

(b) $M=1$

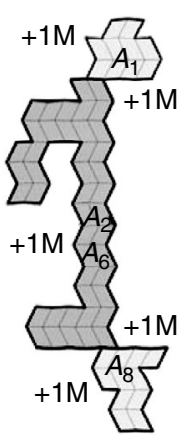

(c) $M=5$

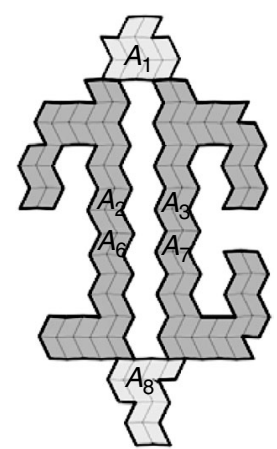

(d) $M=2$

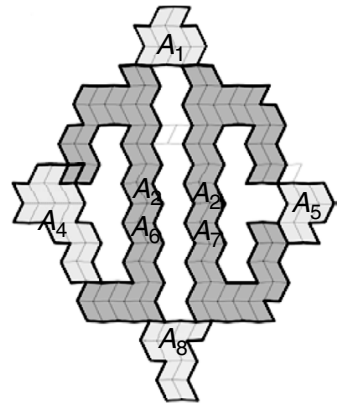

(e) $M=1$

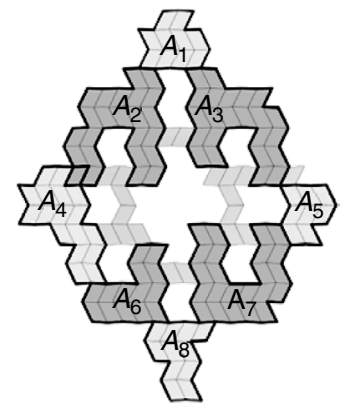

(f) $M=1$
Figure 17. Design process of the Miura Ori mechanism illustrated in Figure 18.

In Figure 17c we see the whole mesh at this stage. There is a closed loop of double-strips areas so that each is connected to the adjacent by one joint or by one face. The loop is comprised of more than three doublestrips areas and there are two consecutive connections made of just one joint. Hence from Remark 2.5 mobility increases.

At this point, Figure 17e, we added the lateral areas $A_{4}$ and $A_{5} . A_{4}$ is connected to its adjacent areas through one joint and through one face. $A_{5}$ is connected through one joint and through one single line strip of one face Globally, they break the series of consecutive single joint connections previously generated and bring the mobility back to one.

Finally (Figure 17e), we added minor connections made of single line strips. These strips obey remarks 
(a)

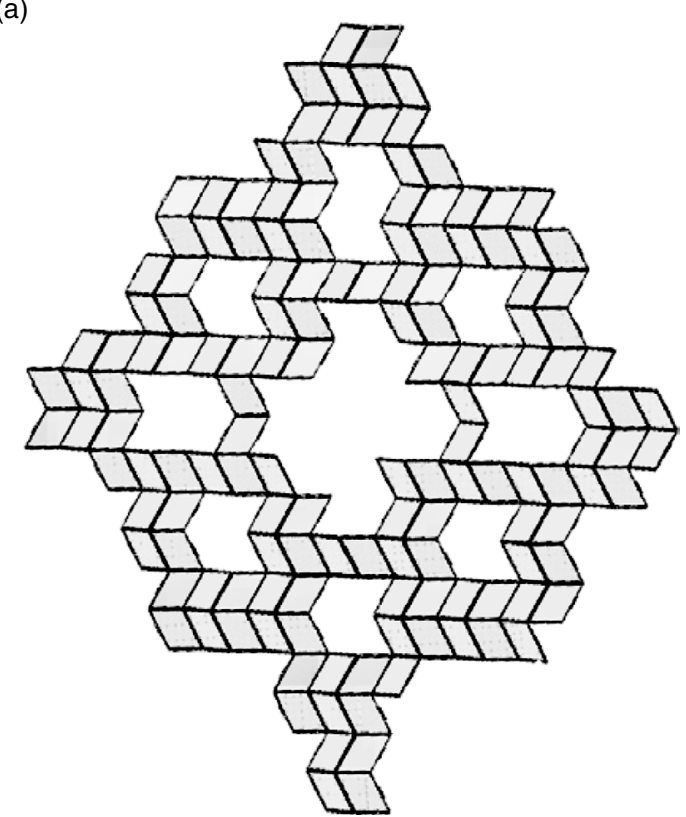

(b)

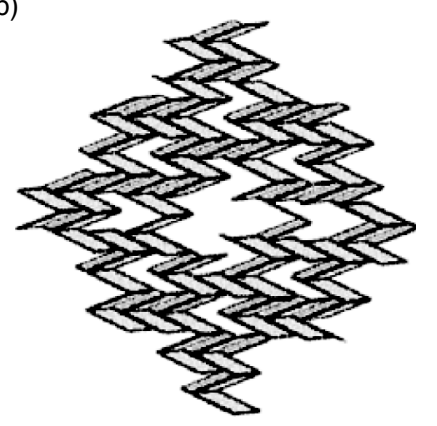

Figure 18. A polyhedral surface with a customized Miura Ori pattern with mobility one. The surface can be designed without the help of any software.

3.4 and 3.6. In fact, they are globally connected twice to a double -line area.

\section{CONCLUSION}

The present work focused on mobility of the Miura Ori mesh, a polyhedral surface that acts as an overconstrained mechanism.

It has shown an easy and effective rule to point out the number of excessive faces that can be removed without affecting the mobility of the system.

Exploiting the presence of excessive faces, the possibility to further customize the mesh has been presented and the rules governing the reciprocal constraint of faces has been shown. In particular, we point out that the design process could be performed without any software. The overall shape could in fact be sketched by hand and then one could develop the connections between faces.

The present paper focuses on the kinematic and design of the mesh, and allows the design of even complex profiles that transmit motion univocally (i.e. with mobility one). Although not of interest here, moving from design to construction it should be noted that such a possibility should be exploited taking care of the structural aspects: a surface with high dissymmetry between branches could be not convenient for the transmission of forces, like in fixed structures.

A further line of investigation would be the opportune use of added degree of freedom. For example, due to the pattern of its faces, the Miura Ori is a developable surface: in the unfolded state it is planar. Other shapes can be achieved changing the geometry of faces, but the efficiency in packaging is heavy reduced. By removing faces opportunely, the mesh could reach instead other surfaces in the unfolded configuration.

\section{ACKNOWLEDGMENTS}

The author ${ }^{1}$ thanks The Scientific \& Technological Research Council of Turkey for its financial support. [Grant number: 2216]

\section{REFERENCES}

[1] Miura, K., Method of packaging and deployment of large membranes in space, in: 31st Congress of the Internatinal Astronautical Federation, IAF, Tokyo, 1980, A 31, 11-19.

[2] Brunner, A., Expansible surface structures, United States patent 3,362,118, 1965.

[3] Miura, K., A note on intrinsic geometry of origami, in: Huzita, H., ed., Proc. of the First International Meeting of Origami Science and Technology, Centro Origami Duffusion, Ferrara, 1989, 239-249.

[4] Hull, T., On the Mathematics of Flat Origamis, Congressus Numerantium, 1994100, 215-224.

[5] Demaine E. D., and O'Rourke, J., Geometric Folding Algorithms. Linkages, Origami, Polyhedra, Cambridge University Press, Cambridge, 2007.

[6] Huffman, D., Curvature and creases. A primer on paper, IEEE Transactions on Computers, 1976, C-25 (10), 1010-1019.

[7] Belcastro, S.M. and Hull, T., Modelling the folding of paper into three dimensions using affine transformations, Linear Algebra and its Applications, 2002, 348, 273-282.

[8] Tachi, T., Freeform Rigid-Foldable Structure using Bidirectionally Flat-Foldable Planar Quadrilateral Mesh, in: Ceccato, C.; Hesselgren, L.; et al., eds., Proc. of Advances in Architectural Geometry, Springer, Vienna, 2010, 87-102.

[9] Tachi, T., Simulation of rigid origami, in: Lang, R., ed., Origami4. Proc. of The Fourth International Conference on Origami in Science, Mathematics, and Education, A K Peters, Ltd., 175-187, 2006.

[10] Stachel, H., A kinematic approach to Kokotsakis meshes, Computer Aided Geometric Design, 2010, 27(6), 428-437.

[11] Dureisseix, D., An Overview of Mechanisms and Patterns with Origami, IJSS, Vol.27, 1, 2012 